\title{
Optical fibre microwire sensors
}

\author{
G. Brambilla*, M. Belal, Y. Jung, Z. Song, F. Xu, T. Newson, D. Richardson \\ ${ }^{a}$ Optoelectronics Research Centre, University of Southampton, Southampton SO17 1BJ, U.K.
}

\begin{abstract}
This paper reviews sensing applications of optical fibre microwires and nanowires. In addition to the usual benefits of sensors based on optical fibres, these sensors are extremely compact and have fast response speeds. In this review sensors will be grouped in three categories according to their morphology: linear sensors, resonant sensors and tip sensors. While linear and resonant sensors mainly exploit the fraction of power propagating outside the microwire physical boundary, tip sensors take advantage of the extreme light confinement to sense chemicals within minute areas.
\end{abstract}

Keywords: Optical fibre microwires, optical fibre sensors, optical fibre tapers,

\section{INTRODUCTION}

Optical fibre micro-/nano-wires (OFM), otherwise known as photonic/optical micro-/nano-wires and optical nano/micro-fibres, are optical fibre tapers with a micrometric or sub-micrometric uniform waist region (fig. 1).

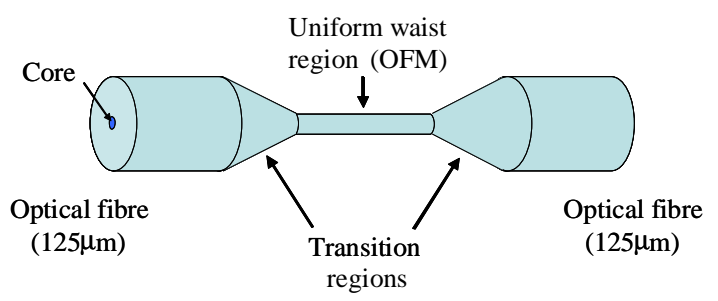

Figure 1. Schematic of an optical fibre micro-/nano-wire (OFM). The micrometric/sub-micrometric uniform waist region is connected by conical transition regions to two optical fiber pigtails.

Recently, OFMs have attracted increasing attention [1], mainly because of their remarkable optical and mechanical properties, which include large evanescent fields, flexibility, robustness, strong confinement, configurability and biocompatibility. OFMs are manufactured tapering optical fibres (fig. 1), thus they are connected by conical transition regions to pigtails, which maintain the original optical fibre size and can be easily connected to other fiberized components. In addition, because of their small diameters, OFMs have a great degree of flexibility and can stand bending radii of few $\mu \mathrm{m}$.

\section{FABRICATION AND PROPERTIES}

\subsection{Fabrication}

OFM manufacturing was carried out mostly using one of the following five methods [1]: flame-brushing [2], modified flame-brushing [3], self-modulated taper-drawing [4], direct draw from bulk [5] and micropipette puller [6]. The "flamebrushing" technique is the most widely used because it uses readily available equipment and it allows for the ultimate control of the taper profile. The "self-modulated taper-drawing" is rather cumbersome, but it has the capability to manufacture minimally invasive OFMs, with radii as small as $r=5 \mathrm{~nm}$. Micropipette puller is a $\mathrm{CO}_{2}$-laser-based commercial equipment which can provide tips with $r \sim 25 \mathrm{~nm}$ and very steep profiles.

\subsection{Guiding properties}

As for conventional optical fibres, in OFMs light is index contrast guided [7]. The OFM guiding properties are determined by the value of $\mathrm{V}$, defined as $V=\kappa \cdot r \cdot N A$ where $\kappa(=2 \pi / \lambda)$ is the propagation constant, $\lambda$ is the

*gb2@ orc.soton.ac.uk; phone +44-23-8059-3588; fax +44-23-8059-3149 
wavelength, $r$ the OFM radius, NA $\left(=\sqrt{n_{O F M}^{2}-n_{\text {surr }}^{2}}\right)$ the OFM numerical aperture, $n_{O F M}$ the OFM refractive index and $n_{\text {surr }}$ the refractive index of the surrounding.

For $V<2.405$, OFMs experience single mode operation and the beam spot size $\omega$ in minimized for $V \sim 2$ [7]. In the $V<2$ region, $\omega$ continuously expands for decreasing $V$ and it becomes orders of magnitude larger than $r$ for $V<0.6$. In this region the fraction of power propagating in the evanescent field $\left(\eta_{E F}\right)$ is considerable and it depends on the refractive index of the surrounding medium and on the ratio $\lambda / r$ [7,8]. Fig. 2 shows the relation between $\eta_{E F}$ and $\lambda / r$ for different surrounding materials, the refractive index of which $\left(\mathrm{n}_{\text {surr }}\right)$ varies between 1 and 1.4: $\eta_{E F}$ increases monotonically for increasing $\lambda / r$ and it reaches $50 \%$ for $\lambda / r \sim 4$ in air (n 1) and for $\lambda / r \sim 2.4$ in water (n 1.33) [8].

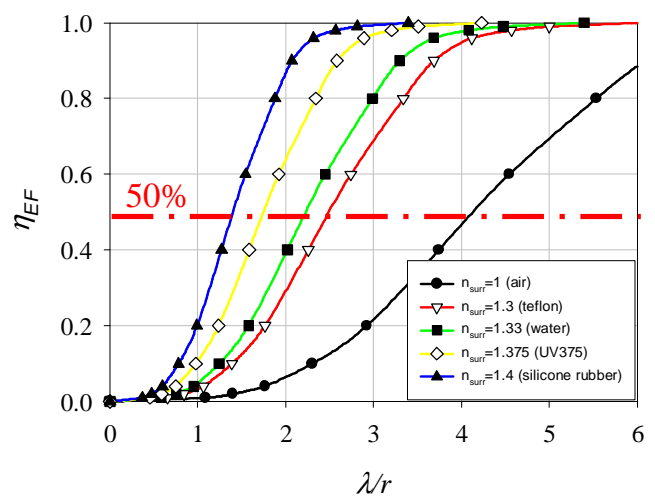

Figure 2. Relation between the fraction of power $\left(\eta_{E F}\right)$ propagating in the evanescent field of an OFM and the normalised wavelength ( $\lambda$ is the wavelength and $r$ the OFM radius) for different surrounding media.

For silica OFMs, maximum confinement corresponds to $\omega \sim \lambda / 3$ in air and $\omega \sim \lambda / 2$ in water. Spot sizes with $\omega \ll \lambda / 3$ cannot be achieved with propagating modes and have been achieved only in metal coated OFMs exploiting the evanescent field along the longitudinal direction [9]. Still, the transmission efficiency $\mathrm{T}_{\varepsilon}$ is generally low: $\mathrm{T}_{\varepsilon} \sim 10^{-8}$ for $r=10 \mathrm{~nm}$ [9].

\section{SENSORS}

OFMs have been deployed in a wide range of sensors, which can be loosely divided into three groups:

1) Confinement; this class of sensors exploits the OFM capability to confine light within small areas and with minimal invasion. This allows for sensing within minute areas and with fast response times.

2) Evanescent field; here the fraction of power propagating outside the OFM physical boundary is used to obtain information on the environment surrounding the OFM.

3) Resonators; high-Q resonators have been manufactured from OFMs in the form of knots, loops and coils. Resonances are extremely sensitive to external perturbations, leading to sensors with high sensitivity.

\subsection{Confinement sensors}

Confinement sensors include two different typologies of sensors exploiting the small OFM size: physical and biochemical sensors. The first group comprises current sensors [10,11], where the small size has been used to measure rapidly changing fields which cannot be measured with conventional optical fibre sensors. Indeed the sensor head comprises a coil with a diameter in the region of $1 \mathrm{~mm}$ and 50 coils: light transit time in the device is in the region of $1 \mathrm{~ns}$ and it allows for the detection of rapidly changing fields (up to $1 \mathrm{MHz}$ ). The second set includes sensors with an extremely small apex cross section (tip sensors), which have been used for minimally invasive point sensing in live cells. As explained in section 2.2, in order to have $\omega<\lambda / 6$ sensors in this category have their lateral surfaces metal coated. The apex was left uncoated (fig. 3) and then functionalized to facilitate covalent immobilization of the molecule to recognise. This group of sensors was originally developed to measure $\mathrm{pH}$ inside single cells [12] and it found applications in the measurement in-situ and in-real-time of a variety of chemical compounds, including potassium, calcium, oxygen, nitrites, chlorides, glutamate, benzopyrene tetrol [13], benzo[a]pyrene, cytochrome c, a cysteine aspartate-dependent protease (Caspase-9), a telomerase, and DNA sequences. 


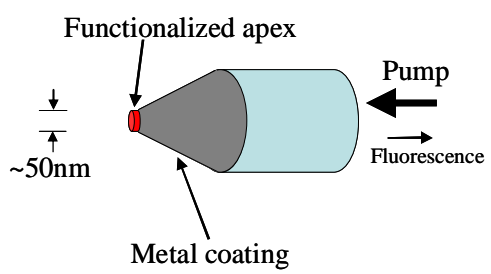

Figure 3. Schematic of an OFM tip sensor. Light injected at the fibre pigtail pumps the functionalised apes, which emits fluorescent light when in proximity of the target compound. Fluorescence is collected via the same pigtail.

\subsection{Evanescent field sensors}

Evanescent field sensors exploit the large fraction of power which propagates outside the ON physical boundary (fig. 2) and thus overlaps with the surrounding fluid. These sensors can exploit the change in the fluid refractive index (refractometric), or its absorption.

A variety of compounds have been measured using absorption changes: some examples include hydrogen [14], $\mathrm{NH}_{3}$ and $\mathrm{NO}_{2}$ [15], gases mixtures, humidity, ammonia, and PTCDA.

Refractometric measurements were used to detect humidity and for microfluidic applications [16]. The humidity sensor was coated with a layer of gelatine, because its refractive index is strongly dependent on the surrounding fluid humidity. A microfluidic sensor was fabricated embedding an OFM in a transparent low refractive index polymer (Sylgard 184) in proximity of a fluidic channel. When the evanescent field of the mode propagating in the OFM overlaps with the microfluidic channel, its transmission loss is strongly dependent on the refractive-index difference between the polymer and the fluid: refractive index changes as small as $5 \cdot 10^{-4}$ have been measured in the fluidic channel.

Silanization and functionalization of the surface in contact with fluids can increase the sensor selectivity and turn a refractometric sensor in a highly specialized chemical sensor.

\subsection{Resonating sensors}

This category includes all sensors exploiting resonant structures; they include two groups of resonators:

1) intrinsic, which can be manufactured from OFMs (like loop [17], knot [4] or microcoil [18] resonators in fig. 4)

2) extrinsic, where the resonator is manufacture by other means (like microspheres, toroids, or capillaries).

Intrinsic resonators have been manufactured exploiting the OFM evanescent field: by coiling an OFM onto itself, modes propagating in two adjacent sections can overlap and couple, producing an extremely compact resonator. The quality factor $Q$ of these resonators can be as high as $10^{6}$.

a)

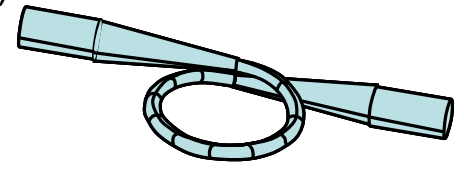

b)

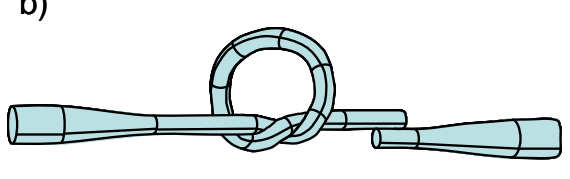

c)

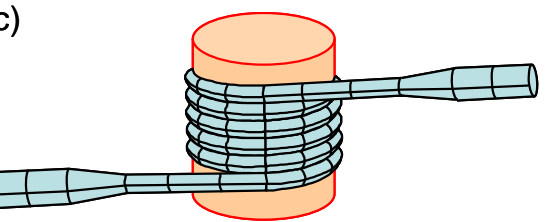

Figure 4. Schematic of intrinsic resonators manufactured from OFMs: a) loop, b) knot and c) microcoil. Knot resonators are generally manufactured from OFMs with a single pigtail, thus they use evanescent coupling for input/output coupling.

Loop and knot sensors include refractometric [19] and temperature sensors [20,21]. Microcoil sensors include refractometric [22,23] and rotation sensors [24]. Because of their 3D geometry, microcoil resonators have an intrinsic channel which can be exploited for microfluidic applications.

Finally OFMs have shown great potentials to insert/extract light from high-Q resonators with coupling efficiencies in excess of $90 \%$ [25]. Evanescent sensing in these types of high-Q resonators has been used to monitor a variety of chemical and biological elements, including DNA [26], streptavidin, single Influenza A virus, single Escherichia Coli bacteria, ethanol and hexane, heavy water, and explosives [27]. 


\section{Acknowledgments}

GB gratefully acknowledges the Royal Society (London, U.K.) for his University Research Fellowship. The authors thank EPSRC for partial financial support.

\section{REFERENCES}

[1] Brambilla, G., "Optical fibre nanowires and microwires: a review”, J. Opt. 12(4), 043001 (2010).

[2] Brambilla, G., Finazzi, V. and Richardson, D.J., "Ultra-low-loss optical fiber nanotapers", Opt. Express 12, 225863, (2004).

[3] Brambilla, G., Koizumi, F., Feng, X., and Richardson, D.J., "Compound-glass optical nanowires", Electron. Lett., 41, 400-2, (2005).

[4] Tong, L.M., Ashcom, J.B., He, S.L., Lou, J.Y., Shen, M.Y., Maxwell, I. and Mazur, E., "Subwavelength-diameter silica wires for low-loss optical waveguiding" Nature, 426, 816-9, (2003).

[5] Tong, L., Hu, L., Zhang, J., Qiu, J., Yang, Q., Lou, J., Shen, Y., He, J., and Ye, Z., "Photonic nanowires directly drawn from bulk glasses" Opt. Express 14(1), 82-87, (2006).

[6] Flaming, K.T., and Brown, D.G., "Advanced Micropipette Techniques for Cell Physiology", Wiley Interscience, (1986).

[7] Tong, L., Lou, J., and Mazur, E., Single-mode guiding properties of subwavelength-diameter silica and silicon wire waveguides, Opt. Express 12, 1025-1035 (2004).

[8] Brambilla, G., "Optical fibre nanotaper sensors", Opt. Fib. Technol., (2011), in press

[9] Hecht, B., Sick, B., Wild, U.P., Deckert, V., Zenobi, R., Martin, O.J.F., and Pohl, D.W., "Scanning near-field optical microscopy with aperture probes: Fundamentals and applications", J. Chem. Phys. 112, 7761-74 (2000).

[10] Belal, M., Song, Z., Jung, Y., Brambilla, G., and Newson, T.P., "Optical fiber microwire current sensor" Opt. Lett. 35, 3045-3047 (2010).

[11]Belal, M., Song, Z., Jung, Y., Brambilla, G., and Newson, T.P., "An interferometric current sensor based on optical fiber micro wires" Opt. Express 18, 19951-19956 (2010).

[12]Tan, W., Shi, Z.Y., Smith, S., Birnbaum, D., Kopelman, R., "Submicrometer intracellular chemical optical fiber sensors" Science 258, 778-781 (1992).

[13] Vo-Dinh, T., Alarie, J.P., Cullum, B.M., Griffin, G.D., "Antibody-based nanoprobe for measurement of a fluorescent analyte in a single cell" Nat. biotechnol. 18(7), 764-7 (2000).

[14] Villatoro, J., and Monzón-Hernández, D., "Fast detection of hydrogen with nano fiber tapers coated with ultra thin palladium layers" Opt. Express 13(13), 5087-92 (2005).

[15] Gu, F., Zhang, L., Yin, X., and Tong, L., Polymer Single-Nanowire Optical Sensors, Nano Lett. 8, 2757-61 (2008).

[16] Polynkin, P., Polynkin, A., Peyghambarian, N., and Mansuripur, M., "Evanescent field-based optical fiber sensing device for measuring the refractive index of liquids in microfluidic channels" Opt. Lett. 30, 1273-5 (2005).

[17] Sumetsky, M., Dulashko, Y. Fini, J.M., Hale, A. and DiGiovanni, D.J. "The Microfiber Loop Resonator: Theory, Experiment, and Application," J. Lightwave Technol. 24(1), 242-250 (2006).

[18] Sumetsky, M., "Optical fiber microcoil resonators", Opt. Express 12(10), 2303-2316 (2004).

[19] Guo, X., and Tong, L. M., "Supported microfiber loops for optical sensing", Opt. Express 16, 14429-34 (2008).

[20] Wu, Y., Rao, Y.-J., Chen, Y.H., and Gong, Y., "Miniature fiber-optic temperature sensors $\square$ based on silica/polymer microfiber knot resonators," Opt. Express 17(20), 18142-7 (2009).

[21]Zeng, X., Wu, Y., Hou, C., Bai, J., and Yang, G., "A temperature sensor based on optical microfiber knot resonator", Opt. Commun. 282, 3817-9 (2009).

[22] Xu, F., Horak, P., and Brambilla, G., "Optical microfiber coil resonator refractometric sensor", Opt. Express, 15, 7888-93 (2007).

[23] Xu, F., and Brambilla, G., "Demonstration of a refractometric sensor based on optical microfiber coil resonator", Appl. Phys. Lett. 92, 101126 (2008).

[24] Scheuer, J., "Fiber microcoil optical gyroscope", Opt. Lett. 34(11), 1630-2 (2009).

[25] Knight, J.C., Cheung, G., Jacques, F., and Birks T.A., "Phase-matched excitation of whispering-gallery-mode resonances by a fiber taper", Opt. Lett. 22(15), 1129-31 (1997).

[26] Vollmer, F., Arnold, S., Braun, D., Teraoka, I., and Libchaber, A., Biophysical J. 85(3), 1974-9 (2003).

[27] Sun, Y., Liu, J., Frye-Mason, G, Ja, S.J., Thompson, A.K., and Fan, X., "Optofluidic ring resonator sensors for rapid DNT vapor detection”, Analyst 134(7), 1386-91 (2009). 